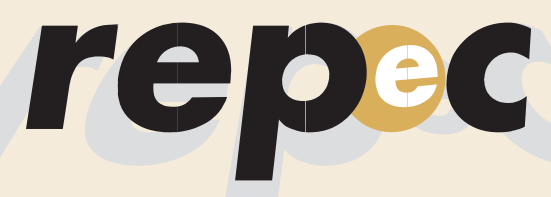

REPeC, Brasília, v. 9, n. 2, art. 2, p. 143-159, abr./jun. 2015 Disponível online em www.repec.org.br DOl: http://dx.doi.org/10.17524/repec.v9i2.1183
Revista de Educação e Pesquisa em Contabilidade Journal of Education and Research in Accounting Revista de Educación e Investigación en Contabilidad

Periódico Trimestral, digital e gratuito publicado pela Academia Brasileira de Ciências Contábeis

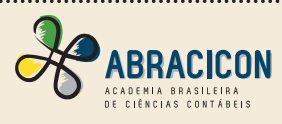

\title{
Pesquisa Bibliométrica e Cienciométrica em Auditoria (2002-2013)
}

\section{Resumo}

Examinamos a produtividade científica sobre auditoria de 2002 a 2013, com base em uma análise bibliométrica/cienciométrica de artigos incluídos na Web of Science do Institute for Scientific Information (ISI), com o objetivo de analisar a evolução temporal da atividade de investigação. A metodologia utilizada foi quantitativa e qualitativa, com análises que englobaram o período de 2002 a 2013 . Os resultados mostram que $60 \%$ das publicações em auditoria estão localizadas na categoria Business Finance e que há um crescimento ao longo do tempo. Como contribuição planearam-se, sistematicamente, as principais características das publicações em auditoria na literatura, por meio de uma análise bibliométrica e cienciométrica para criação do seu estado da arte.

Palavras-Chave: Produção científica, Social Science Citation Index (SSCI), Revisão da literatura.

\author{
Marcelo de Santana Porte \\ Mestre em Administração pela \\ Universidade Federal do Rio Grande \\ do Norte (UFRN) e Doutorando em \\ Contabilidade pela Universidade de \\ Aveiro. Contato: Campus Universitário de \\ Santiago. Aveiro-Portugal. CEP.: 3810-193. \\ E-mail: marcelo_porte@hotmail.com \\ Eliane Silva Sampaio \\ Bacharel em Ciências Contábeis pela \\ Universidade Federal do Maranhão (UFMA) \\ e Mestranda em Contabilidade pela \\ Universidade do Minho. Contato: Campus \\ do Gualtar. Braga-Portugal. CEP.: 4710-057. \\ E-mail: elianessampaio@hotmail.com
}




\section{Introdução}

Embora os investigadores de várias ciências, ao longo dos anos, tenham utilizado técnicas bibliométricas/cienciométricas a fim de conhecerem o que está sendo produzido em determinada área científica, poucos são os estudos encontrados em Contabilidade, especificamente em Auditoria. Como exemplo, pode ser citado o estudo realizado por Moya e Prior (2008), no qual evidenciam a produção científica de uma década em contabilidade nas revistas espanholas e a pesquisa realizada por Neto, Riccio e Sakata (2009), na qual analisam a evolução temporal das publicações na Reunião Anual da Associação Nacional dos Programas de Pós-Graduação em Gestão (Enanpads) realizada no Brasil. Ambos desenvolveram suas pesquisas no período entre 1996-2005.

Recentemente foram realizados estudos em empreendedorismo internacional (Kraus, 2011) e empresa familiar (Chrisman, Kellermanns, Chan \& Liano, 2010; Kraus, Filser, Gotzen \& Harms, 2011), cujo objetivo foi descrever o estado da arte por meio de uma análise de citação, a fim de caracterizar os principais temas, lacunas, bases de pesquisas e tendências futuras na área, demonstrando assim a necessidade de conhecer o que está sendo publicado à luz de uma visão holística para interpretação dos resultados.

Este estudo é importante, no âmbito da pesquisa científica, para que futuros pesquisadores saibam como vêm sendo caracterizadas as publicações em auditoria e quais suas características após o período da promulgação da Lei Sarbanes-Oxley (SOX), além de apresentar uma estrutura metodológica que poderá ser replicada pelos pesquisadores para facilitarem suas futuras pesquisas na Web of Science.

O objetivo do presente estudo é descrever e analisar criticamente a produção em Auditoria, indexadas na Web of Science do Institute for Scientific Information (ISI), no período compreendido entre 2002 e 2013. Dessa forma, pretende-se responder às seguintes inquietações na área de auditoria:

- Em qual categoria as publicações em auditoria estão classificadas na Web of Science?

- Quais as principais fontes de publicação na área?

- Como sua produção tem evoluído ao longo do tempo?

- Como está sendo realizada a parceria em suas publicações?

- Em qual língua as publicações estão sendo realizadas?

- As publicações estão sendo patrocinadas?

- Quais os países que mais publicam?

- Quais as instituições de ensino que mais publicam?

- Quais publicações estão sendo mais utilizadas para base de outras pesquisas?

- Quais os autores/coautores que mais publicam?

- Quais as palavras-chave mais utilizadas nas pesquisas?

A pesquisa terá como base o estudo desenvolvido por Verbeek, Debackere, Luwel e Zimmermann (2002), no qual abordam os principais indicadores que devem ser usados como apoio a um estudo bibliométrico.

Em conformidade com os pensamentos de Verbeek et al. (2002), o estudo que aqui se desenvolve poderá auxiliar pesquisadores juniores e seniores em suas pesquisas futuras, além de suprir a escassez de pesquisas direcionadas a bibliometria e cienciometria em contabilidade, principalmente no tocante às publicações em bases de dados internacionais abrangendo a área de Auditoria.

O trabalho está dividido em cinco seções. Além da sua introdução, na segunda seção, é apresentada a revisão da literatura; na terceira seção, apresenta-se a metodologia empregada; na quarta seção, são apresentados os dados bibliométricos e cienciométricos encontrados; e, posteriormente, é apresentada uma discussão nas conclusões a respeito do que se pode extrair desta investigação, além de indicar limitações e sugerir pesquisas futuras. 


\section{Revisão da Literatura}

Não é de hoje que acadêmicos de várias ciências estão preocupados com o que é publicado em sua área. Dessa forma, há um aumento da frequência do uso da bibliometria/cienciometria nos estudos científicos como uma forma metodológica para se identificar a produção científica dos pares. Encontram-se estudos bibliométricos em várias áreas, tais como: Capital de Risco (Cornelius \& Persson, 2006), Economia (Lee, Cronin, McConnell \& Dean, 2010), Gestão da Cadeia de Suprimentos (Charvet, Cooper \& Gardner, 2008), Governança Corporativa (Durisin \& Puzone, 2009), Marketing (Stremersch \& Verhoef, 2005; Stremersch, Verniers \& Verhoef, 2007), Empresa Familiar (Casillas \& Acedo, 2007), entre outros.

Com o avanço da tecnologia e a existência de diversas fontes de publicações nas mais variadas áreas, fortalece cada vez mais a necessidade de pesquisadores utilizarem recursos tecnológicos em consonância com a metodologia de pesquisa para uma revisão sistemática da literatura e até mesmo para um melhor desenvolvimento de indicadores confiáveis para a análise da atividade científica, uma vez que as bases de dados estão sendo utilizadas como universo amostral em várias pesquisas científicas, a exemplo da Web of Science do Institute for Scientific Information (ISI) (Chang \& Ho, 2010; Duan, 2011; Kostoff, Briggs, Rushenberg, Bowles, Icenhour, Nikodym, Barth \& Pecht, 2007; Kostoff, Tshiteya, Bowles \& Tuunanen, 2006; Machacek \& Kolcunova, 2008; Nerur, Rasheed \& Natarajan, 2008).

Em seu estudo, Verbeek et al. (2002) demonstram como a ciência pode ser mapeada utilizando-se de instrumentos de medição tecnológicos. Vale ressaltar que os mesmos autores ainda relatam que os indicadores quantitativos devem ser complementados com análises qualitativas dos especialistas na área.

É comum uma pesquisa que envolva bibliometria/cienciometria ser vinculada a estudos quantitativos. Contudo, há estudos qualitativos como o de Leal, Almeida e Bortolon (2013) e Bogdan, Iuliana, Valentin e Vasile (2009) que também são usados na literatura, principalmente para (i) explorar como o campo tem evoluído ao longo do tempo, (ii) identificar os grupos de temas de pesquisa que surgiram ao longo do tempo e as relações entre eles, além de (iii) identificar a avaliação de cooperação entre autores e países.

Existem várias formas de aplicabilidade dos estudos bibliométricos/cienciométricos, dentre os quais se destacam:

i) evidenciação das publicações de um país (Butler, 2003; Daraio \& Moed, 2011; Fetscherin, Voss \& Gugler, 2010; Jacobsson \& Rickne, 2004; Jimenez-Contreras, Anegon \& Lopez-Cozar, 2003; Kostoff, Briggs, Rushenberg, Bowles, Icenhour, Nikodym, Barth \& Pecht, 2007; Kostoff, Del Rio, Cortes, Smith, Smith, Wagner, Leydesdorff, Karypis, Malpohl \& Tshiteya, 2005; Kostoff, Johnson, Bowles, Bhattacharya, Icenhour, Nikodym, Barth \& Dodbele, 2007; Kostoff et al., 2006; Sarafoglou, 2006; Schoeneck, Porter, Kostoff \& Berger, 2011);

ii) formação de redes de pesquisa entre universidade-indústria-governo/universidade-indústria/parceria público-privada (PPP) (Abramo, D’Angelo, Di Costa \& Solazzi, 2009, 2011; Hayashi, 2003; Marsilio, Cappellaro \& Cuccurullo, 2011; Park \& Leydesdorff, 2010);

iii) uma área/subárea da ciência (Alfalla-Luque \& Medina-Lopez, 2009; Chabowski, Mena \& Gonzalez-Padron, 2011; Cornelius, Landstrom \& Persson, 2006; Etemad, 2004; Kim \& McMillan, 2008; Ma \& Stern, 2006; Rubin \& Chang, 2003; Serenko \& Bontis, 2013; Talukdar, 2011; Uysal, 2010; Walter, 2010);

iv) as contribuições específicas de um autor (Diamond, 2007; Meyer, Pereira, Persson \& Granstrand, 2004; Uslay, Morgan \& Sheth, 2009);

v) produção científica de um jornal científico ou grupo de jornais (Biemans, Griffin \& Moenaert, 2007; Casey \& McMillan, 2008; Francisco, 2011; Kirchler \& Holzl, 2006; Mazzon \& Hernandez, 2013; McMillan \& Casey, 2007; Ramos-Rodriguez \& Ruiz-Navarro, 2004; Salas \& Sobrevias, 2011; Valacich, Fuller, Schneider \& Dennis, 2006);

vi) livros como agentes de distribuição do conhecimento (Serenko, Bontis \& Moshonsky, 2012);

vii) difusão de uma teoria numa área científica (Weerakkody, Dwivedi \& Irani, 2009). 
Em pesquisa realizada por Groot e Garcia-Valderrama (2006), é visto que "o número de publicações em revistas internacionais de topo é o melhor preditor de resultados da avaliação da revisão de pares", ressaltando o quão significativo é, para o pesquisador, publicar em revistas internacionais, a fim de elevar sua reputação acadêmica e, até mesmo, auxiliá-lo na obtenção de recursos para investimento em pesquisa e desenvolvimento.

Não obstante, órgãos de fomento se valem de tais recursos como um dos indicadores para avaliar a qualidade das publicações, verificando se a pesquisa utiliza-se de fontes de publicações de alta reputação e se as referências da pesquisa possuem um bom fator de impacto (FI), para, assim, efetuarem um apoio financeiro à pesquisa. De acordo com Groot e Garcia-Valderrama (2006), para disponibilizar recursos de apoio financeiro aos programas de pesquisa acadêmica, as fontes patrocinadoras estão avaliando a qualidade de suas publicações e a produtividade de seus colaboradores.

Com a recessão econômica mundial, a economia do conhecimento torna-se um fator preponderante para a elevação do Produto Interno Bruto (PIB) de uma nação. Investimento em Pesquisa e Desenvolvimento $(\mathrm{P} \& \mathrm{D})$ é um dos meios para impulsionar e fortalecer a economia. Contudo, os elevados custos em $\mathrm{P} \& \mathrm{D}$, associados às fontes limitadas de recursos públicos, restringem cada vez mais a alocação de financiamentos para o desenvolvimento científico que são distribuídos conforme o mérito e a capacidade produtiva dos pesquisadores (Abramo, D’Angelo \& Caprasecca, 2009).

Reforçando a informação supracitada, Bengisu e Nekhili (2006) realizaram um estudo no qual procuram alinhar os esforços turcos de previsão tecnológica às atividades internacionais de Ciência e Tecnologia (C\&T). Além disso, visaram obter informações quantitativas para tecnologias prioritárias com o propósito de financiar pesquisas e investir em tecnologia.

\section{Metodologia}

O objetivo da pesquisa centra-se em descrever e analisar criticamente as características da produção científica sobre auditoria na Web of Science desde 2002 - ano da assinatura da Lei Sarbanes-Oxley (SOX) - a 2013 no Social Science Citation Index (SSCI) por meio de uma análise bibliométrica e cienciométrica.

O período da amostra inicia-se em 2002, em decorrência do impacto mundial sofrido pela auditoria após os escândalos envolvendo os relatórios financeiros da Eron, que culminaram com a criação da SOX. O objetivo dessa Lei é garantir a criação de mecanismos de auditoria e segurança confiáveis nas empresas, incluindo regras para a criação de comitês encarregados de supervisionar suas atividades e operações, de modo a mitigar os riscos do negócio, evitar a ocorrência de fraudes ou assegurar que haja meios de identificá-las quando ocorrem, garantindo a transparência na gestão das empresas.

Foi utilizada a palavra "audit " no campo Topic (envolve título, resumo e palavras-chave) com a limitação do período da pesquisa de 1900 a 2013 na base de dados de citação no SSCI. Após tal procedimento, o refinamento apresentou 40.140 resultados. O próximo processo foi a realização da seleção dos tipos de documentos, refinando para os grupos Article e Review, diminuindo, dessa forma, para 34.670 resultados. Posteriormente, foi selecionada a área de pesquisa de Business Economics, pois, com base em um pré-teste realizado, é a área que possui mais características com o tema proposto, gerando um indicador de 4.572 resultados. Contudo, é válido salientar que, apesar de ter sido efetuado o refino para a área supracitada, outras áreas aparecem pelo motivo de que a mesma publicação pode estar classificada em mais de uma área. Por esse motivo, para manter a melhor abrangência da pesquisa, não foram efetuadas as exclusões das áreas que não foram refinadas, justamente pelo fato de as suas publicações estarem em outra área. Caso fosse feita a exclusão, iriam ser excluídas, também, as publicações de uma das áreas desejadas para o refino. Vale ressaltar que os presentes dados aqui coletados foram atualizados até 10 de janeiro de 2014.

O próximo procedimento foi selecionar as publicações de 2002 a 2013, total de 2.480 resultados, e transportá-las para o programa EndNote X5 para que fosse realizada uma Revisão Sistemática da Literatura com objetivo de evidenciar os resultados encontrados exclusivamente na área de auditoria. Realizada 
esta etapa, foi possível encontrar 2.394 publicações em auditoria após a exclusão dos outlines. Em seguida, essas referências foram transportadas para o programa Nvivo10, a fim de elaborar uma base de dados específica sobre o assunto para que fosse realizada uma abordagem metodológica quantitativa e qualitativa com base em análises estatísticas, matemática e de conteúdo.

Os resultados obtidos servirão para que futuros pesquisadores saibam quais os principais autores de auditoria que estão na base de dados Web of Science, além de informações, como: quais instituições estão a efetuar estudos na área; quais fontes de publicações, autores e países que mais publicam; quais palavras-chave mais usadas, entre outros.

\section{Resultados}

Com base nos pressupostos metodológicos utilizados para o período de 2002 a 2013, após a Revisão Sistemática da Literatura, foram encontradas 86 publicações, do total de 2.480 publicações que não possuem vínculo com a área de Auditoria. Logo, foram utilizadas 2.394 publicações na presente pesquisa (Tabela 1).

Tabela 1

Perfil das publicações analisadas

\begin{tabular}{|c|c|c|}
\hline Perfil & $\mathbf{P}$ & $\mathbf{F 1}(\cong)$ \\
\hline $\begin{array}{l}\text { Publicações de } 2002 \text { a } 2013 \text { na Web of Science (filtro uso do termo "audit*") na área } \\
\text { de Business Economics }\end{array}$ & 2.480 & $100 \%$ \\
\hline Outlines (artigos excluídos por não haver vínculo com a área de auditoria) & 86 & $3,47 \%$ \\
\hline $\begin{array}{l}\text { Publicações selecionadas após análise de conteúdo vinculadas à área de } \\
\text { Auditoria }\end{array}$ & 2.394 & $96,53 \%$ \\
\hline Publicações analisadas nessa pesquisa & 2.394 & $100 \%$ \\
\hline
\end{tabular}

$\mathrm{P}=$ Número de publicações.

Fonte: dados da pesquisa

As publicações em auditoria concentram-se, basicamente, em quatro grandes categorias classificadas pela Web of Science: Business Finance, Economics, Management e Business. Contudo, a que possui maior destaque é a primeira por conter mais da metade de representatividade das publicações na área; as outras três possuem um equilíbrio na faixa dos $19 \%$ a $22 \%$ dos resultados encontrados (Tabela 2).

Tabela 2

Distribuição das categorias da Web of Science por quantidade de publicações com no mínimo 3\%

\begin{tabular}{|c|c|c|}
\hline Categoria da Web of Science & $\mathbf{P}$ & $\mathbf{F}_{1}(\cong)$ \\
\hline Business Finance & 1.436 & $60 \%$ \\
\hline Economics & 524 & $22 \%$ \\
\hline Management & 507 & $21 \%$ \\
\hline Business & 449 & $19 \%$ \\
\hline Ethics & 114 & $5 \%$ \\
\hline Public Administration & 75 & $3 \%$ \\
\hline
\end{tabular}

$\mathrm{P}=$ Número de publicações.

Fonte: dados da pesquisa 
Conforme Tabela 3, o jornal/revista que mais publica em auditoria é o Auditing-a Journal of Practice \& Theory (299; 12,5\%), seguido pelo Accounting Review (162; 6,8\%) e Contemporary Accounting Rese$\operatorname{arch}(158 ; 6,6 \%)$. O Top 13 jornais/revistas soma mais da metade das publicações na área $(1.233,51,5 \%)$, demonstrando a importância que seus editores dão ao referido tema. A Accounting Review possui também outro destaque, que é a relação de vezes que seus trabalhos foram citados (3.577) e sem autocitações (3.191), possuindo, assim, a segunda maior média de citações $(22,08)$, ficando atrás apenas do Journal of Accounting Research, com uma média de 31,20 citações por publicação.

Tabela 3

Distribuição das fontes de publicações por Dados Gerais

\begin{tabular}{|c|c|c|c|c|c|c|}
\hline Fonte de Publicação & Sigla & $\mathbf{P}$ & $F_{1}(\cong)$ & C1 & C2 & $\bar{x}$ \\
\hline Auditing-a Journal of Practice \& Theory & AJPT & 299 & $12,5 \%$ & 2.263 & 1.531 & 7,57 \\
\hline Accounting Review & AR & 162 & $6,8 \%$ & 3.577 & 3.191 & 22,08 \\
\hline Contemporary Accounting Research & CAR & 158 & $6,6 \%$ & 1.982 & 1.738 & 12,54 \\
\hline Journal of Business Ethics & JBE & 105 & $4,4 \%$ & 668 & 609 & 6,36 \\
\hline Accounting Organizations and Society & A LOS & 80 & $3,3 \%$ & 1.043 & 925 & 13,04 \\
\hline Corporate Governance-an International Review & CGIR & 62 & $2,6 \%$ & 386 & 345 & 6,23 \\
\hline Journal of Accounting and Public Policy & JAPP & 60 & $2,5 \%$ & 166 & 142 & 2,77 \\
\hline Journal of Accounting Research & JAR & 54 & $2,3 \%$ & 1.685 & 1.638 & 31,20 \\
\hline Accounting Horizons & $\mathrm{AH}$ & 54 & $2,3 \%$ & 162 & 145 & 3 \\
\hline Journal of Accounting \& Economics & JAE & 54 & $2,3 \%$ & 2.214 & 2.143 & 41 \\
\hline African Journal of Business Management & AJBM & 52 & $2,2 \%$ & 28 & 24 & 0,54 \\
\hline Accounting and Finance & $\mathrm{AF}$ & 50 & $2,1 \%$ & 150 & 126 & 3 \\
\hline European Accounting Review & EAR & 43 & $1,8 \%$ & 176 & 164 & 4,09 \\
\hline Outras 330 fontes de publicações & - & 1.161 & $48,5 \%$ & - & - & - \\
\hline Total & & 2.394 & $100 \%$ & - & - & - \\
\hline
\end{tabular}

$\mathrm{P}=$ Número de publicações; $\mathrm{C} 1$ = Número de citações; $\mathrm{C} 2$ = Número de citações sem autocitações; $\bar{x}=$ Média de citações por item.

Fonte: dados da pesquisa

Há muitas fontes de publicações que possuem produções com vinculação à área de auditoria (343). Todavia, mais da metade das publicações $(61,5 \%)$ podem ser vistas em apenas 13 jornais/revistas (Tabela 3), das quais apenas nove possuem Fator de Impacto (FI) > 1 (Tabela 4). O jornal/revista que se destaca é o Journal of Accounting \& Economics por possuir FI próximo a quatro e por ser a terceira fonte de publicação com maior Eigenfactor (EF), ficando atrás apenas do Journal of Bussiness Ethics e Accounting Review, além de possuir maior Article Influence (AI). 
Tabela 4

Top 13 das fontes de publicações por Fator de Impacto (FI) e Eigenfactor (EF) e Article Influence (AI)

\begin{tabular}{lcccc}
\hline \multicolumn{1}{c}{ Fonte de Publicação } & Sigla & FI & EF & AI \\
\hline Journal of Accounting \& Economics & JAE & $\mathbf{3 , 9 1 2}$ & 0,00741 & $\mathbf{2 , 4 5 3}$ \\
\hline Accounting Review & AR & 2,319 & 0,00795 & 1,474 \\
\hline Journal of Accounting Research & JAR & 2,192 & 0,00703 & 2,210 \\
\hline Accounting Organizations and Society & AOS & 1,867 & 0,00364 & 1,028 \\
\hline Contemporary Accounting Research & CAR & 1,564 & 0,00348 & 1,094 \\
\hline Corporate Governance-an International Review & CGIR & 1,400 & 0,00164 & 0,364 \\
\hline Accounting Horizons & AH & 1,288 & 0,00117 & não informado \\
\hline Journal of Business Ethics & JBE & 1,253 & $\mathbf{0 , 0 1 3 9 5}$ & 0,450 \\
\hline Auditing-a Journal of Practice \& Theory & AJPT & 1,015 & 0,00110 & 0,483 \\
\hline Accounting and Finance & AF & 0,875 & 0,00065 & 0,192 \\
\hline Journal of Accounting and Public Policy & JAPP & 0,770 & 0,00100 & não informado \\
\hline European Accounting Review & EAR & 0,654 & 0,00102 & 0,453 \\
\hline African Journal of Business Management & AJBM & não informado & não informado & não informado \\
\hline
\end{tabular}

Fator de Impacto (FI) de 2012; Eigenfactor (EF) e Article Influence (Al) atualizados até 10/01/2014.

Fonte: dados da pesquisa

De acordo com a Tabela 5, os autores de quase 1/3 das publicações (758) do Top 13 das fontes de publicações concentram-se em apenas seis jornais/revistas (AOS, CAR, CGIR, AH, JBE e AJPT) com FI entre ]1;2]. O resultado ainda melhora se forem vistas as fontes de publicações com FI entre ]1;3] com oito fontes de publicações (JAE, AR, JAR, AOS, CAR, CGIR, AH, JBE e AJPT), as quais agrupam 974 publicações, sendo responsáveis por, praticamente, $40 \%$ das publicações em auditoria.

Tabela 5

Distribuição do Fator de Impacto (FI) do Top 13 das fontes de publicações

\begin{tabular}{cccc}
\hline Fator de Impacto em $\mathbf{2 0 1 2}$ & $\mathbf{J}$ & $\mathbf{P}$ & $\mathbf{F}_{\mathbf{i}}(\cong)$ \\
\hline $0<\mathrm{FI} \leq 1$ & 3 & 153 & $6,4 \%$ \\
\hline $1<\mathrm{FI} \leq 2$ & $\mathbf{6}$ & $\mathbf{7 5 8}$ & $\mathbf{3 1 , 7 \%}$ \\
\hline $2<\mathrm{FI} \leq 3$ & 2 & 216 & $9 \%$ \\
\hline $3<\mathrm{FI}$ & 1 & 54 & $2,3 \%$ \\
\hline Não informado & 1 & 52 & $2,2 \%$ \\
\hline Não analisado (N/A) & 330 & 1.161 & $48,5 \%$ \\
\hline Total & $\mathbf{3 4 3}$ & $\mathbf{2 . 3 9 4}$ & $\mathbf{1 0 0 \%}$ \\
\hline
\end{tabular}

$\mathrm{J}=$ Número de fontes de publicações; $\mathrm{P}$ = Número de publicações.

Fonte: dados da pesquisa

Percebe-se que, ao longo do tempo, houve uma tendência de mais jornais/revistas publicarem sobre auditoria, com destaque para o ano de 2011, conforme dados da Figura 1, com 311 trabalhos publicados, haja vista que possivelmente o número de publicações submetidas também tenha aumentado pelo fato de a Lei Sarbanes-Oxley (SOX) ter sido criada em 2002.

A pesquisa não pode explicar o motivo do aumento das publicações. Contudo, é válido lembrar que, em 2005, as empresas de capital aberto da União Europeia foram obrigadas a adotar as normas da International Financial Reporting Standards (IFRS), demonstrando ser, possivelmente, um indicador do aumento nas produções em auditoria. É importante desenvolver estudos que verifiquem qual o motivo real do crescimento de tais publicações, e dessa forma, complementem a informação aqui relatada. 


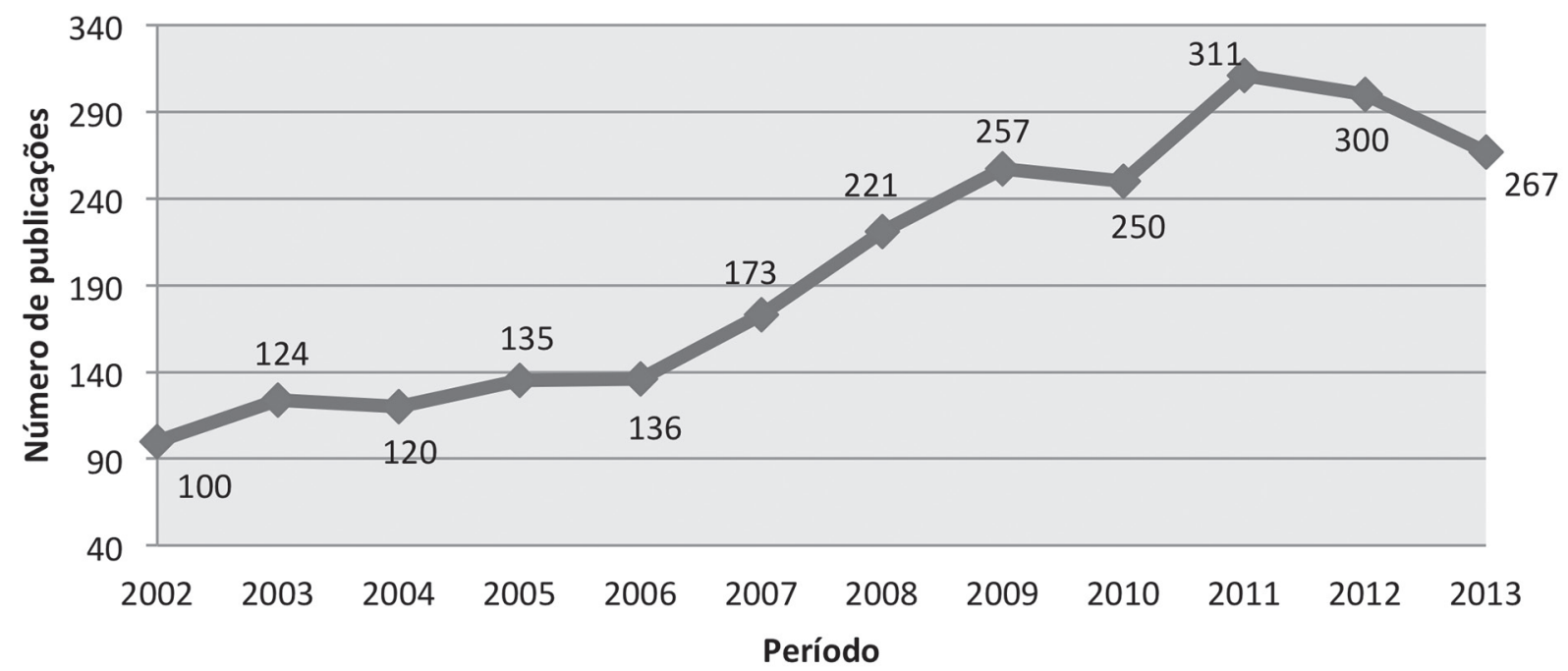

Figura 1. Distribuição das publicações por ano

Fonte: dados da pesquisa.

Com base na Tabela 6, observa-se que 79\% das publicações aqui encontradas foram realizadas em parceria, demonstrando assim a necessidade de se trabalhar em grupo para a obtenção de resultados mais expressivos. Contudo, há um destaque entre as parcerias com dois e três autores, por representarem aproximadamente $69 \%$ dos estudos.

Ainda na Tabela 6, podemos detectar que, notoriamente, os trabalhos são publicados em inglês (96\%). Posteriormente, com uma representatividade bem menor, as publicações são escritas em alemão, espanhol, russo e francês. Infelizmente, os resultados demonstram que apenas $1 \%$ dos estudos ocorridos obtiveram fontes de financiamento para suas pesquisas, confirmando que governos/empresas não estão muitos dispostos a investir em novos estudos na área de auditoria.

Tabela 6

Perfil da parceria dos autores, língua da publicação e fomento da pesquisa

\begin{tabular}{lcc}
\hline \multicolumn{1}{c}{ Perfil } & $\mathbf{P}$ & $\mathbf{F}_{\mathbf{i}}(\cong)$ \\
\hline $\mathbf{N}^{\mathbf{0}}$ de publicações analisadas & $\mathbf{2 . 3 9 4}$ & $\mathbf{1 0 0 \%}$ \\
\hline $\mathrm{N}^{\circ}$ de autores por publicações & & $\mathbf{2 1 \%}$ \\
\hline Publicações com um autor & 495 & $\mathbf{3 6 \%}$ \\
\hline Publicações com dois autores & $\mathbf{8 5 3}$ & $\mathbf{3 3 \%}$ \\
\hline Publicações com três autores & 797 & $9 \%$ \\
\hline Publicações com quatro & 205 & $2 \%$ \\
\hline Publicações com mais que quatro autores & 44 & $\mathbf{9 6 \%}$ \\
\hline Língua das publicações & & $1,2 \%$ \\
\hline Inglês & $\mathbf{2 . 2 9 8}$ & $0,9 \%$ \\
\hline Alemão & 28 & $0,7 \%$ \\
\hline Espanhol & 21 & $0,5 \%$ \\
\hline Russo & 16 & $0,8 \%$ \\
\hline Francês & 12 & $1 \%$ \\
\hline Outras línguas & 19 & $99 \%$ \\
\hline Fomento da pesquisa & & 23 \\
\hline Financiada & 2.371 & \\
\hline Não informado sobre financiamento & & \\
\hline
\end{tabular}

$\mathrm{P}=$ Número de publicações.

Fonte: dados da pesquisa 
No total, 70 países realizaram publicações que envolvem a área de Auditoria, sendo que, em 35 registros, não informado o país de afiliação para a sua publicação, havendo destaque para as publicações americanas com mais da metade das publicações em Auditoria, seguidos por Austrália, Canadá, Inglaterra, China, Alemanha, entre outros (Tabela 7).

Tabela 7

Top 10 dos países com publicações

\begin{tabular}{ccc}
\hline Países & $\mathbf{P}$ & $\mathbf{F i}(\cong)$ \\
\hline EUA & $\mathbf{1 . 2 3 4}$ & $\mathbf{5 1 , 5 \%}$ \\
\hline Austrália & 213 & $8,9 \%$ \\
\hline Canadá & 199 & $8,3 \%$ \\
\hline Inglaterra & 177 & $7,4 \%$ \\
\hline China & 145 & $6,1 \%$ \\
\hline Alemanha & 83 & $3,5 \%$ \\
\hline Taiwan & 82 & $3,4 \%$ \\
\hline Espanha & 76 & $3,2 \%$ \\
\hline Holanda & 74 & $3,1 \%$ \\
\hline Nova Zelândia & 59 & $2,5 \%$ \\
\hline Outros 60 países & 657 & $27,4 \%$ \\
\hline
\end{tabular}

$\mathrm{P}=$ Número de publicações.

Fonte: dados da pesquisa

De acordo com os resultados, mais da metade das publicações são americanas e 7 (sete) entre as 11 instituições que mais publicaram em Auditoria também são americanas, havendo destaque para a Florida International University System, com 130 publicações (Tabela 8).

Tabela 8

Distribuição das instituições (organizations-enhanced) com publicações (autores e coautores)

\begin{tabular}{|c|c|c|c|}
\hline Instituições (Organizations-Enhanced) & País & $\mathbf{P}$ & $\mathrm{Fi}(\cong)$ \\
\hline Florida International University System & EUA & 130 & $5,4 \%$ \\
\hline University of California System & EUA & 55 & $2,3 \%$ \\
\hline University of New South Wales & Austrália & 54 & $2,3 \%$ \\
\hline University of Wisconsin System & EUA & 52 & $2,2 \%$ \\
\hline Pennsylvania Commonwealth System of Higher Education Pcshe & EUA & 51 & $2,1 \%$ \\
\hline Hong Kong Polytechnic University & Hong Kong & 42 & $1,8 \%$ \\
\hline Northeastern University & EUA & 42 & $1,8 \%$ \\
\hline Nanyang Technological University & Cingapura & 39 & $1,6 \%$ \\
\hline $\begin{array}{l}\text { Nanyang Technological University National Institute of Education Nie } \\
\text { Singapore }\end{array}$ & Cingapura & 39 & $1,6 \%$ \\
\hline Florida International University & EUA & 38 & $1,6 \%$ \\
\hline University of Wisconsin Madison & EUA & 38 & $1,6 \%$ \\
\hline
\end{tabular}

$\mathrm{P}=$ Número de publicações.

Fonte: dados da pesquisa

O artigo que possui o maior número de citações é o "Theorizing change: The role of professional associations in the transformation of institutionalized fields", dos autores Greenwood, Suddaby e Hinings, com 437 citações ocorridas desde 2002 e também com a maior média de citações por ano 33,32 (Tabela 9). 
Tabela 9

Top 15 dos trabalhos mais citados

\begin{tabular}{|c|c|c|c|c|c|}
\hline $\mathbf{N}^{\circ}$ & $\begin{array}{l}\text { Vezes } \\
\text { Citado }\end{array}$ & $\bar{x}$ & Autores & Título do Artigo & Ano \\
\hline 1 & 437 & 33,62 & $\begin{array}{l}\text { Greenwood, R; Suddaby, } \\
\text { R; Hinings, CR }\end{array}$ & $\begin{array}{l}\text { Theorizing change: The role of professional associations in } \\
\text { the transformation of institutionalized fields }\end{array}$ & 2002 \\
\hline 2 & 355 & 27,31 & Klein, A & $\begin{array}{l}\text { Audit committee, board of director characteristics, and } \\
\text { earnings management }\end{array}$ & 2002 \\
\hline 3 & 238 & 18,31 & $\begin{array}{l}\text { Frankel, RM; Johnson, MF; } \\
\text { Nelson, KK }\end{array}$ & $\begin{array}{l}\text { The relation between auditors' fees for nonaudit services } \\
\text { and earnings management }\end{array}$ & 2002 \\
\hline 4 & 217 & 21,70 & Ball, R; Shivakumar, L & $\begin{array}{l}\text { Earnings quality in UK private firms: comparative loss } \\
\text { recognition timeliness }\end{array}$ & 2005 \\
\hline 5 & 214 & 17,83 & Ball, R; Robin, A; Wu, JS & $\begin{array}{l}\text { Incentives versus standards: properties of accounting } \\
\text { income in four East Asian countries }\end{array}$ & 2003 \\
\hline 6 & 187 & 15,58 & $\begin{array}{l}\text { Ashbaugh, H; LaFond, R; } \\
\text { Mayhew, BW }\end{array}$ & $\begin{array}{l}\text { Do nonaudit services compromise auditor independence? } \\
\text { Further evidence }\end{array}$ & 2003 \\
\hline 7 & 184 & 14,15 & Mitton, $T$ & $\begin{array}{l}\text { A cross-firm analysis of the impact of corporate } \\
\text { governance on the East Asian financial crisis }\end{array}$ & 2002 \\
\hline 8 & 170 & 13,08 & $\begin{array}{l}\text { DeFond, ML; } \\
\text { Raghunandan, K; } \\
\text { Subramanyam, KR }\end{array}$ & $\begin{array}{l}\text { Do non-audit service fees impair auditor independence? } \\
\text { Evidence from going concern audit opinions }\end{array}$ & 2002 \\
\hline 9 & 159 & 12,23 & Morrison, EW & $\begin{array}{l}\text { Newcomers' relationships: The role of social network ties } \\
\text { during socialization }\end{array}$ & 2002 \\
\hline 10 & 137 & 11,42 & Joh, SW & $\begin{array}{l}\text { Corporate governance and firm profitability: evidence } \\
\text { from Korea before the economic crisis }\end{array}$ & 2003 \\
\hline 11 & 135 & 13,50 & Agrawal, A; Chadha, S & Corporate governance and accounting scandals & 2005 \\
\hline 12 & 128 & 11,64 & $\begin{array}{l}\text { Palmrose, ZV; Richardson, } \\
\text { VJ; Scholz, S }\end{array}$ & $\begin{array}{l}\text { Determinants of market reactions to restatement } \\
\text { announcements }\end{array}$ & 2004 \\
\hline 13 & 125 & 15,62 & Olken, Benjamin A. & $\begin{array}{l}\text { Monitoring corruption: Evidence from a field experiment } \\
\text { in Indonesia }\end{array}$ & 2007 \\
\hline 14 & 125 & 9,62 & $\begin{array}{l}\text { Nelson, MW; Elliott, JA; } \\
\text { Tarpley, RL }\end{array}$ & $\begin{array}{l}\text { Evidence from auditors about managers' and auditors' } \\
\text { earnings management decisions }\end{array}$ & 2002 \\
\hline 15 & 124 & 10,33 & $\begin{array}{l}\text { Xie, B; Davidson, WN; } \\
\text { DaDalt, PJ }\end{array}$ & $\begin{array}{l}\text { Earnings management and corporate governance: the } \\
\text { role of the board and the audit committee }\end{array}$ & 2003 \\
\hline
\end{tabular}

$\bar{x}=$ Média de citações por ano.

Fonte: dados da pesquisa

É válido salientar que não foi analisado o conteúdo das citações, tornando-se possível que futuras pesquisas possam abordar essa vertente para que tenhamos um parâmetro de como foram ocorridas essas citações.

O autor que mais possui publicações em auditoria é o Kannan Raghunandan, com 26 publicações, sendo citado 525 vezes e possuindo a segunda melhor média de citações por trabalho citado, $(20,19)$, ficando atrás apenas de Jere R. Francis, com uma média de 26,76 citações (Tabela 10). Da mesma forma, quer nos artigos mais citados, quer nas publicações por autor, não se levou em consideração o teor das citações, sendo necessário que ocorra em pesquisas futuras. 
Tabela 10

Top 10 dos autores e coautores com maior produção

\begin{tabular}{llcccc}
\multicolumn{1}{c}{ Autor } & P & Vezes Citado & $\begin{array}{c}\text { Vezes citado sem } \\
\text { autocitações }\end{array}$ & $\overline{\boldsymbol{X}}$ & H-index \\
\hline Raghunandan, Kannan & 26 & 525 & $\mathbf{5 0 1}$ & $\mathbf{2 0 , 1 9}$ & 10 \\
\hline Knechel, W. Robert & 22 & 201 & 175 & 9,14 & 7 \\
\hline Gul, Ferdinand A. & 19 & 197 & 184 & 10,37 & 8 \\
\hline Francis, Jere R. & 17 & 455 & 430 & $\mathbf{2 6 , 7 6}$ & $\mathbf{1 1}$ \\
\hline Krishnan, Jayanthi & 17 & 246 & 223 & 14,47 & 6 \\
\hline Tan, Hun-Tong & 17 & 194 & 179 & 11,41 & 8 \\
\hline Bedard, Jean C. & 16 & 267 & 245 & 16,69 & 9 \\
\hline Trotman, Ken T. & 16 & 140 & 126 & 8,75 & 7 \\
\hline Johnstone, Karla M. & 15 & 172 & 160 & 11,47 & 7 \\
\hline Rama, Dasaratha V. & 15 & 173 & 168 & 11,53 & 7 \\
\hline
\end{tabular}

$\mathrm{P}=$ Número de publicações; $\bar{x}=$ Média de citações por ano.

Fonte: dados da pesquisa

Observamos na Figura 2, popularmente conhecida como nuvem de termos, as palavras mais repetidas entre as publicações encontradas. Vale salientar que da base de dados formada por 2.394 publicações, cerca de 2.275 apresentaram palavras-chave em seus trabalhos, correspondendo a $95 \%$ do total. Notamos, pelas palavras destacadas em fonte maior, os termos - chave comuns que circundam a temática trabalhada e, obrigatoriamente, o termo audit se destaca por ter sido usado como modo de filtro para a pesquisa. Contudo os resultados dão ênfase também a outros nove termos: management, earnings, quality, performance, corporate, governance, auditor risk, information.

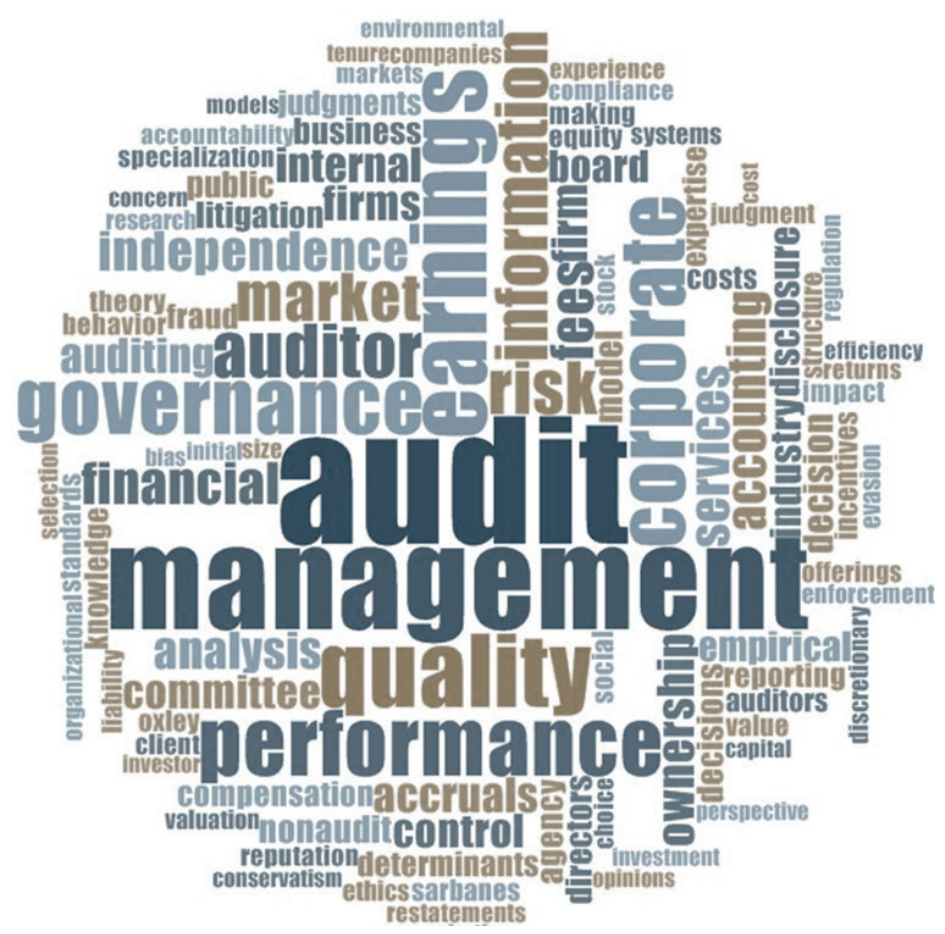

Figura 2. Nuvem de termos das palavras-chave

Fonte: Dados da pesquisa 
A base de dados formada por 2.394 publicações possui 23.162 citações e, sem a autocitação, têm-se 13.010. Vale destacar que em mais de 10 mil trabalhos houve citação das publicações analisadas nessa pesquisa. Excetuando-se as autocitações, esse número é reduzido para 9.210 pesquisas, originando uma média de 9,68 citações por publicação e um H-Index de 66 (Tabela 11).

Tabela 11

Perfil H-index das publicações analisadas

\begin{tabular}{|c|c|}
\hline Perfil & $\mathbf{P}$ \\
\hline Publicações analisadas nessa pesquisa (a). & 2.394 \\
\hline Vezes que as publicações analisadas foram citadas (b). & 23.162 \\
\hline $\begin{array}{l}\text { Vezes que as publicações analisadas foram citadas sem autocitação (self-citations) entre as próprias } \\
\text { publicações analisadas. }\end{array}$ & 13.010 \\
\hline Quantidade de publicações que citaram as publicações analisadas. & 10.695 \\
\hline $\begin{array}{l}\text { Quantidade de publicações que citaram as publicações analisadas sem autocitação (self-citations) dentre as } \\
\text { próprias publicações analisadas. }\end{array}$ & 9.210 \\
\hline Média de vezes que as publicações foram citadas $(b \div a)$. & 9,68 \\
\hline H-index. & 66 \\
\hline
\end{tabular}

$\mathrm{P}=$ Número de publicações.

Fonte: dados da pesquisa

Os resultados aqui encontrados planeiam, por meio de uma visão global, as publicações em auditoria com base em um estudo bibliométrico e cienciométrico realizado na Web of Science.

\section{Conclusão}

Mediante análise dos resultados, pode-se verificar que houve expressivo crescimento quantitativo da produção acadêmica de Auditoria em revistas indexadas na Web of Science por ser uma base de dados reconhecida no meio acadêmico. Isso demostra um fator positivo. Todavia alguns pontos merecem reflexão mais aprofundada. Ainda que o número de trabalhos publicados tenha crescido, saindo de 100 publicações em 2002 e atingindo o seu melhor resultado em 2011, com 311 publicações realizadas, esses números sugerem que é necessário que pesquisadores continuem publicando na área de Auditoria. Para que isso ocorra, é necessário que editores abram mais espaços nas revistas de contabilidade e gestão, lancem edições especiais ou elaborarem periódicos exclusivos para a área de Auditoria. Ainda que as publicações em Auditoria dividam espaços nas revistas com outros temas de contabilidade e, até mesmo, com temas de administração, por que está ocorrendo, então, essa redução nas publicações em auditoria a partir de 2011? Será isso uma tendência? Será que os pesquisadores não estão mais interessados na área de auditoria ou o seu espaço diminuiu nas revistas após esse período? Essas são reflexões válidas porque os resultados refletem uma leve queda nas publicações em auditoria após o seu crescimento significativo a partir de 2002. Embora os dados levantados não proporcionem evidências empíricas sobre o assunto, supomos que, em decorrência da adoção das normas internacionais de contabilidade em muitos países e por terem sido notificados escândalos empresariais em nível mundial ao longo desse período, as publicações em Auditoria estejam encontrando sua estabilidade.

Verificamos que $82 \%$ das publicações em Auditoria estão classificadas nas categorias Business Finance e Economics na base de dados da Web of Science, o que possibilita que pesquisadores utilizem as presentes categorias como filtros no seu sistema a fim de facilitar a procura por artigos na área. O estudo também revela que a revista Auditing-a Journal of Practice \& Theory vem contribuindo de forma mais expressiva no tocante ao número de publicações na área. Isso evidência a importância de revistas especializadas na área para que pesquisadores tenham um maior espaço para as suas publicações. 
Uma constatação feita é que não é tarefa fácil desenvolver pesquisas sozinho, pois os resultados demonstram que 79\% das produções em Auditoria são feitas em parceria e que a língua inglesa é predominante nas publicações da área, além de supreendentemente ser verificado que apenas $1 \%$ das produções na área possuíram fomento em suas pesquisas. Tais resultados sugerem a importância de os autores estarem envolvidos em bases de pesquisa para que ocorra uma maior troca de conhecimento entre os pares e, assim, possam ter sucesso nas aprovações de suas publicações. A partir destas constatações, surgem-nos inquietações: por que a insignificância no tocante ao fomento de pesquisa na área? Será que esse reflexo ocorre também com pesquisas aqui no Brasil?

Outro dado importante é que as universidades americanas dominam as publicações em auditora, com mais da metade das publicações na área, havendo destaque para a Florida International University System, com 130 publicações indexadas. Seria interessante que futuros pesquisadores fizessem uma comparação entre a abordagem das publicações americanas na área de Auditora para que sejam identificados parâmetros iguais e divergentes em seus estilos de pesquisa.

Um fato que nos chamou a atenção nos resultados é que existe publicações com números de citações altíssimos, com destaque para a publicação Theorizing change: The role of professional associations in the transformation of institutionalized fields, dos autores Greenwood, Suddaby e Hinings, com 437 citações ocorridas desde 2002 e também com a maior média de citações por ano $(33,32)$. Outro destaque é para o autor Kannan Raghunandan, com 26 publicações, sendo citado 525 vezes e possuindo a segunda melhor média de citações por trabalho citado, $(20,19)$. Seria interessante entender o porquê do destaque dessas publicações e dos autores na área? O que as publicações mais citadas estão trazendo de inovador? Será que essas citações são realmente todas positivas?

Por fim, verificamos que as palavras management, earnings, quality, performance, corporate, governance, auditor risk, information estão sendo mais usadas como classificação das pesquisas como palavras-chave. Dessa forma, pesquisadores podem utilizar esses termos para realizarem filtros em suas pesquisas na base de dados da Web of Science. Seria interessante que futuras pesquisas evidenciassem se esses termos realmente possuem alguma simetria com as principais temáticas das publicações na área de Auditoria.

Para futuras pesquisas, sugere-se a comparação das publicações encontradas na Web of Science com as publicações da Scopus e a realização de análises comparativas das publicações brasileiras com as produções dos países anglo-saxões, salientando a causa de eventuais diferenças que podem estar relacionadas com o tempo de harmonização das normas internacionais de contabilidade em cada país, com aspectos econômicos e culturais, além das demais inquietações supracitadas ao longo da conclusão.

A principal contribuição deste trabalho é que ele planeia, sistematicamente, as características fundamentais das publicações em Auditoria na literatura estendida por meio de uma análise bibliométrica e cienciométrica na área, favorecendo, assim, o trabalho prévio de muitos pesquisadores, que podem iniciar seus estudos a partir dos dados aqui apresentados.

\section{Referências}

Abramo, G., D’Angelo, C. A. \& Caprasecca, A. (2009). Allocative efficiency in public research funding: Can bibliometrics help? Research Policy, 38(1), pp. 206-215. doi: 10.1016/j.respol.2008.11.001.

Abramo, G., D’Angelo, C. A., Di Costa, F. \& Solazzi, M. (2009). University-industry collaboration in Italy: A bibliometric examination. Technovation, 29(6-7), pp. 498-507. doi: 10.1016/j.technovation.2008.11.003.

Abramo, G., D’Angelo, C. A., Di Costa, F. \& Solazzi, M. (2011). The role of information asymmetry in the market for university-industry research collaboration. Journal of Technology Transfer, 36(1), pp. 84-100. doi: 10.1007/s10961-009-9131-5. 
Alfalla-Luque, R. \& Medina-Lopez, C. (2009). Supply Chain Management: Unheard of in the 1970s, core to today's company. Business History, 51(2), pp. 202-221. doi: 10.1080/00076790902726558.

Bengisu, M. \& Nekhili, R. (2006). Forecasting emerging technologies with the aid of science and technology databases. Technological Forecasting and Social Change, 73(7), pp. 835-844. doi: 10.1016/j. techfore.2005.09.001.

Biemans, W., Griffin, A. \& Moenaert, R. (2007). Twenty years of the Journal of product innovation management: History, participants, and knowledge stock and flows. Journal of Product Innovation Management, 24(3), pp. 193-213. doi: 10.1111/j.1540-5885.2007.00245.x.

Bogdan, I., Iuliana, I., Valentin, D. \& Vasile, G. (2009). The quality management of the services of scientometric quantification of the research of the members of the university community deployed within the "e-univroscient" concept. Amfiteatru Economic, 11(26), pp. 429-440. doi: 105897/ AJBM2014.7607

Butler, L. (2003). Explaining Australia's increased share of ISI publications - the effects of a funding formula based on publication counts. Research Policy, 32(1), pp. 143-155. doi: 10.1016/s00487333(02)00007-0.

Casey, D. L. \& McMillan, G. S. (2008). Identifying the "invisible colleges" of the industrial \& labor relations review: a bibliometric approach. Industrial \& Labor Relations Review, 62(1), pp. 126-132. doi: 10.5897/AJBM2014.7607

Casillas, J. \& Acedo, F. (2007). Evolution of the intellectual structure of family business literature: A bibliometric study of FBR. Family Business Review, 20(2), pp. 141-162. doi: 10.1111/j.1741-6248.2007.00092.x.

Chabowski, B. R., Mena, J. A. \& Gonzalez-Padron, T. L. (2011). The structure of sustainability research in marketing, 1958-2008: a basis for future research opportunities. Journal of the Academy of Marketing Science, 39(1), pp. 55-70. doi: 10.1007/s11747-010-0212-7.

Chang, C. C. \& Ho, Y. S. (2010). Bibliometric analysis of financial crisis research. African Journal of Business Management, 4(18), pp. 3898-3910. doi: 10.1007/s11192-013-1014-8

Charvet, F. F., Cooper, M. C. \& Gardner, J. T. (2008). The intellectual structure of supply chain management: A bibliometric approach. Journal of Business Logistics, 29(1), pp. 47-73. doi: 10.1002/j.21581592.2008

Chrisman, J. J., Kellermanns, F. W., Chan, K. C. \& Liano, K. (2010). Intellectual Foundations of Current Research in Family Business: An Identification and Review of 25 Influential Articles. Family Business Review, 23(1), pp. 9-26. doi: 10.1177/0894486509357920.

Cornelius, B., Landstrom, H. \& Persson, O. (2006). Entrepreneurial studies: The dynamic research front of a developing social science. Entrepreneurship Theory and Practice, 30(3), pp. 375-398. doi: 10.1111/j.1540-6520.2006.00125.x.

Cornelius, B. \& Persson, O. (2006). Who's who in venture capital research. Technovation, 26(2), pp. 142150. doi: 10.1016/j.technovation.2005.05.009.

Daraio, C. \& Moed, H. F. (2011). Is Italian science declining? Research Policy, 40(10), pp. 1380-1392. doi: 10.1016/j.respol.2011.06.013

Diamond, A. M. (2007). Thriving at Amazon: How Schumpeter lives in books today. Econ Journal Watch, 4(3), pp. 338-344. doi: 10.1017/S1053837209990307

Duan, C. H. (2011). Mapping the intellectual structure of modern technology management. Technology Analysis \& Strategic Management, 23(5), pp. 583-600. doi: 10.1080/09537325.2011.565672. 
Durisin, B. \& Puzone, F. (2009). Maturation of Corporate Governance Research, 1993-2007: An Assessment. Corporate Governance-an International Review, 17(3), pp. 266-291. doi: 10.1111/j.1467-8683.2009.00739.x.

Etemad, H. (2004). E-commerce: the emergence of a field and its knowledge network. International Journal of Technology Management, 28(7-8), pp. 776-800. doi: 10.1504/ijtm.2004.005783.

Fetscherin, M., Voss, H. \& Gugler, P. (2010). 30 Years of foreign direct investment to China: An interdisciplinary literature review. International Business Review, 19(3), pp. 235-246. doi: 10.1016/j.ibusrev.2009.12.002.

Francisco, E. D. (2011). RAE-Eletronica: exploration of archive in the light of bibliometrics, geoanalysis and social network. Rae-Revista de Administração de Empresas, 51(3), pp. 280-306. doi: 10.1590/ S0034-75902011000300008.

Groot, T. \& Garcia-Valderrama, T. (2006). Research quality and efficiency - An analysis of assessments and management issues in Dutch economics and business research programs. Research Policy, 35(9), pp. 1362-1376. doi: 10.1016/j.respol.2006.07.002.

Hayashi, T. (2003). Effect of R\&D programmes on the formation of university-industry-government networks: comparative analysis of Japanese R\&D programmes. Research Policy, 32(8), pp. 1421-1442. doi: 10.1016/s0048-7333(02)00158-0.

Jacobsson, S. \& Rickne, A. (2004). How large is the Swedish 'academic' sector really? A critical analysis of the use of science and technology indicators. Research Policy, 33(9), pp. 1355-1372. doi: 10.1016/j. respol.2004.09.002.

Jimenez-Contreras, E., Anegon, F. D. \& Lopez-Cozar, E. D. (2003). The evolution of research activity in Spain - The impact of the National Commission for the Evaluation of Research Activity (CNEAI). Research Policy, 32(1), pp. 123-142. doi: 10.1016/s0048-7333(02)00008-2.

Kim, J. \& McMillan, S. J. (2008). Evaluation of Internet advertising research - A bibliometric analysis of citations from key sources. Journal of Advertising, 37(1), pp. 99-112. doi: 10.2753/joa00913367370108 .

Kirchler, E. \& Holzl, E. (2006). Twenty-five years of the Journal of Economic Psychology (1981-2005): A report on the development of an interdisciplinary field of research. Journal of Economic Psychology, 27(6), pp. 793-804. doi: 10.1016/j.joep.2006.07.001.

Kostoff, R. N., Briggs, M. B., Rushenberg, R. L., Bowles, C. A., Icenhour, A. S., Nikodym, K. F., Barth, R.B. \& Pecht, M. (2007). Chinese science and technology - Structure and infrastructure. Technological Forecasting and Social Change, 74(9), pp. 1539-1573. doi: 10.1016/j.techfore.2007.02.008.

Kostoff, R. N., Del Rio, J., Cortes, H. D., Smith, C., Smith, A., Wagner, C., Leydesdorff, L. \& Tshiteya, R. (2005a). The structure and infrastructure of Mexico's science and technology. Technological Forecasting and Social Change, 72(7), pp. 798-814. doi: 10.1016/j.techfore.2005.02.001.

Kostoff, R. N., Johnson, D., Bowles, C. A., Bhattacharya, S., Icenhour, A. S., Nikodym, K., Barth, R.B. \& Dodbele, S. (2007). Assessment of India's research literature. Technological Forecasting and Social Change, 74(9), pp. 1574-1608. doi: 10.1016/j.techfore.2007.02.009.

Kostoff, R. N., Tshiteya, R., Bowles, C. A. \& Tuunanen, T. (2006). The structure and infrastructure of Finnish research literature. Technology Analysis \& Strategic Management, 18(2), pp. 187-220. doi: 10.1080/09537320600624105.

Kraus, S. (2011). State-of-the-art current research in international entrepreneurship: A citation analysis. African Journal of Business Management, 5(3), pp. 1020-1038. doi: 10.5897/AJBM10.1159. 
Kraus, S., Filser, M., Gotzen, T. \& Harms, R. (2011). Family firms - On the state-of-the-art of business research. Betriebswirtschaftliche Forschung Und Praxis, 63(6), pp. 587-605. doi: $10.1177 / 0894486511435355$

Leal, R. P. C., Almeida, V. D. E. \& Bortolon, P. M. (2013). Brazilian scientific production in finance in the period 2000-2010. Rae-Revista de Administração de Empresas, 53(1), pp. 46-55. doi: 10.1590/ S0034-75902013000100005.

Lee, F. S., Cronin, B. C., McConnell, S. \& Dean, E. (2010). Research Quality Rankings of Heterodox Economic Journals in a Contested Discipline. American Journal of Economics and Sociology, 69(5), pp. 1409-1452. doi: 10.1111/j.1536-7150.2010.00751.x.

Ma, C. B. \& Stern, D. I. (2006). Environmental and ecological economics: A citation analysis. Ecological Economics, 58(3), pp. 491-506. doi: 10.1016/j.ecolecon.2005.07.023.

Machacek, M. \& Kolcunova, E. (2008). Hirsch index and rankings of Czech economists. Politicka Ekonomie, 56(2), pp. 229-241. doi: 10.5897/AJBM2014.7607

Marsilio, M., Cappellaro, G. \& Cuccurullo, C. (2011). The intellectual structure of research into PPPS: A bibliometric analysis. Public Management Review, 13(6), pp. 763-782. doi: 10.1080/14719037.2010.539112.

Mazzon, J. A. \& Hernandez, J. M. D. (2013). Brazilian scientific production in marketing in the period 2000-2009. Rae-Revista de Administração de Empresas, 53(1), pp. 67-80. doi: 10.1590/S0034-75902013000100007.

McMillan, G. S. \& Casey, D. L. (2007). Research note: Identifying the invisible colleges of the British journal of industrial relations: A bibliometric and social network approach. British Journal of Industrial Relations, 45(4), pp. 815-828. doi: 10.1111/j.1467-8543.2007.00645.x.

Meyer, M., Pereira, T. S., Persson, O. \& Granstrand, O. (2004). The scientornetric world of Keith Pavitt - A tribute to his contributions to research policy and patent analysis. Research Policy, 33(9), pp. 14051417. doi: 10.1016/j.respol.2004.07.008.

Moya, S. \& Prior, D. (2008). Who publish in Spanish accounting jornals? A bibliometric analysis 19962005. Revista Espanola De Financiacion Y Contabilidad-Spanish Journal of Finance and Accounting, 37(138), pp. 353-374. doi: 10.1080/02102412.2008.10779648.

Nerur, S. P., Rasheed, A. A. \& Natarajan, V. (2008). The intellectual structure of the strategic management field: An author co-citation analysis. Strategic Management Journal, 29(3), pp. 319-336. doi: 10.1002/smj.659.

Neto, O. R. D., Riccio, E. L. \& Sakata, M. C. G. (2009). Ten years of accounting research in Brazil: analysis with papers of ENANPADs from 1996 to 2005. Rae-Revista De Administracao De Empresas, 49(1), pp. 62-73. doi: 10.1590/S0034-75902009000100008.

Park, H. W. \& Leydesdorff, L. (2010). Longitudinal trends in networks of university-industry-government relations in South Korea: The role of programmatic incentives. Research Policy, 39(5), pp. 640-649. doi: 10.1016/j.respol.2010.02.009.

Ramos-Rodriguez, A. R. \& Ruiz-Navarro, J. (2004). Changes in the intellectual structure of strategic management research: A bibliometric study of the Strategic Management Journal, 1980-2000. Strategic Management Journal, 25(10), pp. 981-1004. doi: 10.1002/smj.397.

Rubin, R. M. \& Chang, C. F. (2003). A bibliometric analysis of health economics articles in the economics literature: 1991-2000. Health Economics, 12(5), pp. 403-414. doi: 10.1002/hec.802.

Salas, O. A. \& Sobrevias, E. O. (2011). Retrospective analysis of the Spanish Journal of Finance and Accounting (1985-2011): Authors, subjects, citations and quality perception. Revista Espanola De Financiacion Y Contabilidad-Spanish Journal of Finance and Accounting, 40(152), pp. 699-721. doi: 10.5897/ AJBM2014.7607 
Sarafoglou, N. (2006). How to measure productivity and how productive are Swedish professors in economics? Research evaluation by using quantitative and qualitative indicators. Ekonomiska Samfundets Tidskrift, 59(2), pp. 95-103.

Schoeneck, D. J., Porter, A. L., Kostoff, R. N. \& Berger, E. M. (2011). Assessment of Brazil's research literature. Technology Analysis \& Strategic Management, 23(6), pp. 601-621. doi: 10.1080/09537325.2011.585029.

Serenko, A. \& Bontis, N. (2013). Global ranking of knowledge management and intellectual capital academic journals: 2013 update. Journal of Knowledge Management, 17(2), pp. 307-326. doi: $10.1108 / 13673271311315231$.

Serenko, A., Bontis, N. \& Moshonsky, M. (2012). Books as a knowledge translation mechanism: citation analysis and author survey. Journal of Knowledge Management, 16(3), pp. 495-511. doi: $10.1108 / 13673271211238797$.

Stremersch, S. \& Verhoef, P. C. (2005). Globalization of authorship in the marketing discipline: Does it help or hinder the field? Marketing Science, 24(4), pp. 585-594. doi: 10.1287/mksc.1050.0152.

Stremersch, S., Verniers, I. \& Verhoef, P. C. (2007). The quest for citations: Drivers of article impact. Journal of Marketing, 71(3), pp. 171-193. doi: 10.1509/jmkg.71.3.171.

Talukdar, D. (2011). Patterns of Research Productivity in the Business Ethics Literature: Insights from Analyses of Bibliometric Distributions. Journal of Business Ethics, 98(1), pp. 137-151. doi: 10.1007/ s10551-010-0539-5.

Uslay, C., Morgan, R. E. \& Sheth, J. N. (2009). Peter Drucker on marketing: an exploration of five tenets. Journal of the Academy of Marketing Science, 37(1), pp. 47-60. doi: 10.1007/s11747-008-0099-8.

Uysal, O. O. (2010). Business Ethics Research with an Accounting Focus: A Bibliometric Analysis from 1988 to 2007. Journal of Business Ethics, 93(1), pp. 137-160. doi: 10.1007/s10551-009-0187-9.

Valacich, J. S., Fuller, M. A., Schneider, C. \& Dennis, A. R. (2006). Issues and opinions - Publication opportunities in premier business outlets: How level is the playing field? Information Systems Research, 17(2), pp. 107-125. doi: 10.1287/isre.1060.0089.

Verbeek, A., Debackere, K., Luwel, M. \& Zimmermann, E. (2002). Measuring progress and evolution in science and technology - I: The multiple uses of bibliometric indicators. International Journal of Management Reviews, 4(2), pp. 179-211. doi: 10.1111/1468-2370.00083.

Walter, F. (2010). The profile of the papers published on Custos e @gronegocio on line. Custos E Agronegocio, 6(3), pp. 156-175. doi: 10.5897/AJBM2014.7607

Weerakkody, V., Dwivedi, Y. K. \& Irani, Z. (2009). The diffusion and use of institutional theory: a cross-disciplinary longitudinal literature survey. Journal of Information Technology, 24(4), pp. 354-368. doi: 10.1057/jit.2009.16. 